\title{
Graft interpenetrating polymer networks of urethane-modified bismaleimide and epoxy (I): mechanical behavior and morphology
}

\author{
K.H. Hsieh ${ }^{\mathrm{a}, *}$, J.L. Han ${ }^{\mathrm{b}}$, C.T. Yu ${ }^{\mathrm{a}}$, S.C. Fu ${ }^{\mathrm{a}}$ \\ ${ }^{a}$ Department of Chemical Engineering, National Taiwan University, Taipei 106, Taiwan, ROC \\ ${ }^{\mathrm{b}}$ Department of Chemical Engineering, National I-Lan Institute of Technology, I-Lan, Taiwan, ROC
}

Received 26 April 2000; received in revised form 21 August 2000; accepted 21 August 2000

\begin{abstract}
Polyurethanes (PU) based on poly(butylene adipate) [PU(PBA)] and poly(oxypropylene) [PU(PPG)] polyols are employed as a graft agent to prepare interpenetrating polymer networks of urethane-modified bismaleimide (UBMI) and the diglycidyl ether of bisphenol A (Ep) (UBMI/Ep graft-IPNs). The UBMI is introduced and partially grafted to the epoxy by PU graft agents, and then simultaneous bulk polymerization technique is used to prepare the graft-IPNs.

All the PU graft agents were characterized by infrared (IR). The tensile strength of both the UBMI/Ep graft-IPNs with PU(PBA) and PU(PPG) graft agent systems increased to a maximum value with increasing UBMI content in the system and then decreased with further increasing the UBMI content. For both kinds of PU with various molecular weight in the UBMI/Ep graft-IPNs, the Izod impact strength increased with the UBMI contents increasing. The better compatibility of PU(PBA)-based UBMI/Ep graft-IPNs led to higher impact strength. On the contrary, the fracture energy $\left(G_{\mathrm{IC}}\right.$ value) of the resultant UBMI/Ep graft-IPN showed that the UBMI/Ep graft-IPN with PU(PPG) graft agent had much higher $G_{I C}$ value than that with PU(PBA) graft agent. The morphology of this IPN with PU(PBA) graft agent exhibited a homogeneous one-phase system, while the UBMI/Ep graft-IPN with PU(PPG) graft agent showed a two-phase morphology with the UBMI particles dispersed in the epoxy matrix. (C) 2000 Elsevier Science Ltd. All rights reserved.
\end{abstract}

Keywords: Urethane-modified bismaleimide; Graft interpenetrating polymer networks; Morphologies

\section{Introduction}

Owing to the mechanical properties of polymer materials with interpenetrating polymer network structures are superior to those of ordinary polymers [1-4]. In particular, the maximum tensile strength of interpenetrating polymer networks (IPNs) is much higher than that of the constituent polymers. Therefore, many valuable systems have been made by taking advantage of the IPN structure. Our previous works [5-9] have attempted to enhance the mechanical properties by synthesizing a series of graft IPNs (graft-IPNs) of polyurethane (PU) and epoxy.

Epoxy resins have rather high glass-transition temperature $T_{\mathrm{g}}$, and they are quite hard and brittle in nature. In 1994, Hsieh et al. $[10,11]$ proposed that the urethane-modified bismaleimide (UBMI) rubber have excellent thermal stability and mechanical properties. Processes for preparing IPNs can mainly be classified into sequential and simultaneous polymerization [12]. In order to improve the mechanical properties of epoxy resin, the UBMI is introduced and partially grafted to

\footnotetext{
* Corresponding author. Tel.: +886-2-2362-7688; fax: +886-2-23627688.

E-mail address: khhsieh@ccms.ntu.edu.tw (K.H. Hsieh).
}

the epoxy by PU graft agents, and then simultaneous bulk polymerization technique is used to prepare the graft-IPNs. PU graft agents based on both polyester-type and polyethertype polyols, with various molecular weights, are selected to prepare the UBMI/Ep graft-IPNs used in this work. The stress-strain property, Izod impact strength, and fracture energy $\left(G_{\mathrm{IC}}\right)$ of these UBMI/Ep graft-IPNs are investigated. In addition, the relations between their mechanical behavior and morphologies are discussed.

\section{Experimental}

\subsection{Material}

The materials used and their designations are listed in Table 1. Polyols (PPG400, PPG1000, PPG2000, PBA700, PBA1000, and PBA2000) and epoxy (DGEBA) were continuously stirred and degassed overnight at $60^{\circ} \mathrm{C}$ in the flask before they were used.

\subsection{Preparation of $P U$ prepolymer}

PU prepolymers were prepared by reacting $4,4^{\prime}$-diphenyl methane diisocyanate (MDI) with polyols. MDI (2 


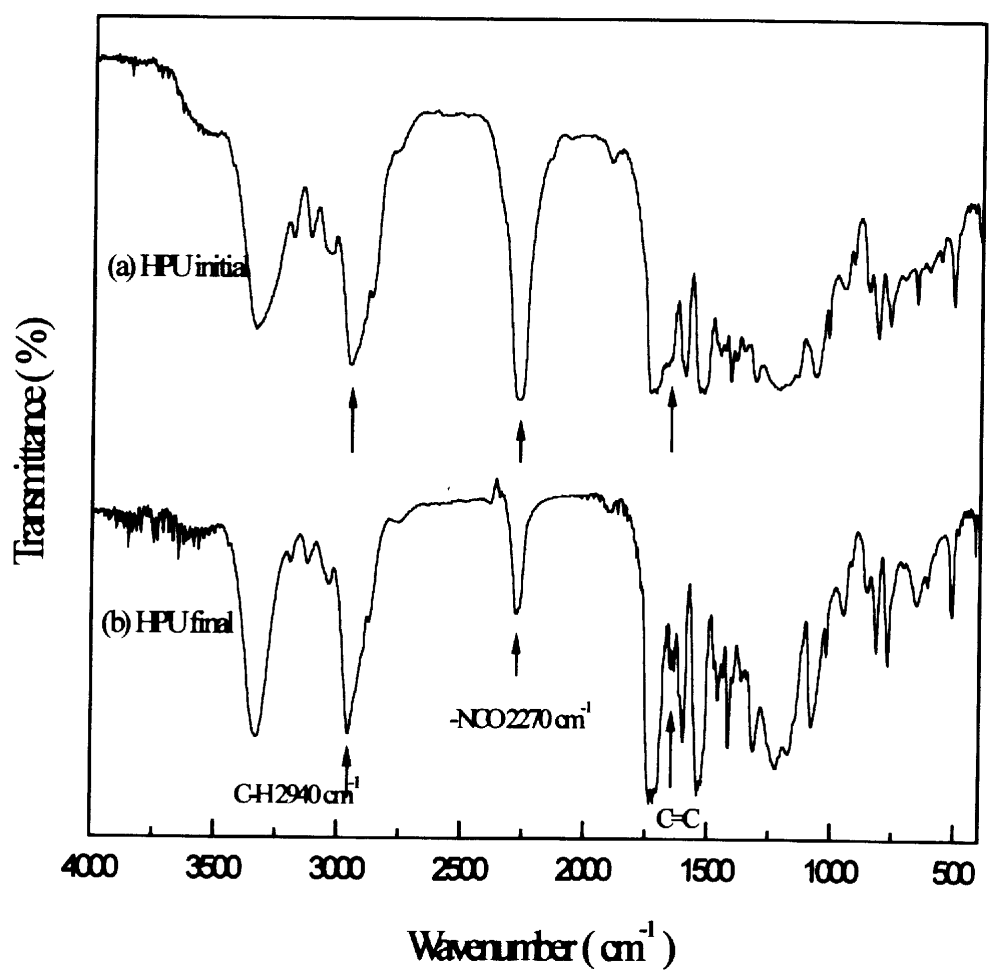

Fig. 1. FT-IR spectra during the relation of PU(PBA700) prepolymer with the hydroxyl group of HEMA: (a) initial; (b) final.

equivalent weight) was initially poured in a reaction kettle and heated to melting state. Polyols in proper amount (1 equivalent weight) were then poured into the reaction kettle. The mixture was vigorously agitated by a mechanical stirrer. The reaction occurred under dry nitrogen atmosphere at a temperature of approximately $68^{\circ} \mathrm{C}$. The isocyanate content of the reaction mixture was determined by using di-n-butyl amine titration method [13]. The reaction was curtailed when the isocyanate content reached a theoretical value.

\subsection{Preparation of $P U$ graft agent}

The PU graft agent was synthesized by a two step reaction process. First, the condensation reaction of 2-hydroxyethyl methacrylate (HEMA) and NCO-terminated PU prepolymer was performed according to the following reaction: where $\mathrm{R}$ is the urethane-terminated polyether or polyester diols.

The PU prepolymers were mixed with HEMA, with a molar ratio of $1: 1$, in toluene (solvent) and was dropped $1 \mathrm{wt} \%$ (based HEMA) triethylamine (TEA) catalyst. The reaction occurred under dry nitrogen atmosphere at $68^{\circ} \mathrm{C}$. During this period, samples were drawn for infrared (IR) analysis. The reaction was curtailed when the intensity of the $-\mathrm{NCO}$ absorption peak at $2270 \mathrm{~cm}^{-1}$ was reduced to a constant intensity. Secondly, the solution was poured into the epoxy resin (DGEBA, DER331) after removing the TEA and the residual isocyanate group $(-\mathrm{NCO})$ of the solution was then reacted with the secondary hydroxyl group of the DGEBA resin $(\mathrm{NCO} / \mathrm{OH}=1: 1)$ to form the urethane linkage. In addition, the PU graft agent was formed. The reaction was performed according to<smiles>C=C(C)C(=O)OCC[OH+]</smiles>

2-hydroxyethyl methacrylate PU prepolymer

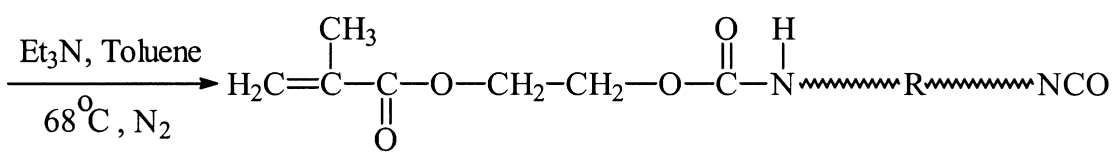


the following reaction:<smiles>C=C(C)C(=O)OCCOC(=O)N[C@H](C)C(=O)O</smiles><smiles></smiles>

During this period, samples were drawn for IR analysis. The reaction was curtailed when the $-\mathrm{NCO}$ absorption peak at $2270 \mathrm{~cm}^{-1}$ disappeared. A slightly viscous liquid was obtained after removing the solvent by vacuum. Herein, the PU graft agent based on PPG and PBA is used as a coupling agent for the graft IPNs of UBMI and epoxy.

\subsection{Preparation of UBMI [10,11]}

The UBMI was synthesized by the condensation reaction of maleic anhydride and NCO-terminated PU prepolymer according to the following reaction:
The reaction took place under dry nitrogen atmosphere at a temperature of approximately $68^{\circ} \mathrm{C}$. The NCO-terminated PU prepolymer, dissolved in DMF, was added to the solution of maleic anhydride and tertiary amine catalyst (TEA) at $68^{\circ} \mathrm{C}$. The start of the reaction was observed by the evolution of $\mathrm{CO}_{2}$ gas. During this period, samples were drawn for IR analysis. The reaction was stopped when the NCO absorption peak at $2270 \mathrm{~cm}^{-1}$ and the $\mathrm{C}=\mathrm{O}$ (of acid anhydride) absorption peak at $1848 \mathrm{~cm}^{-1}$ disappeared. To remove the DMF, the solution was poured into methanol and the precipitate was washed with acetone periodically.

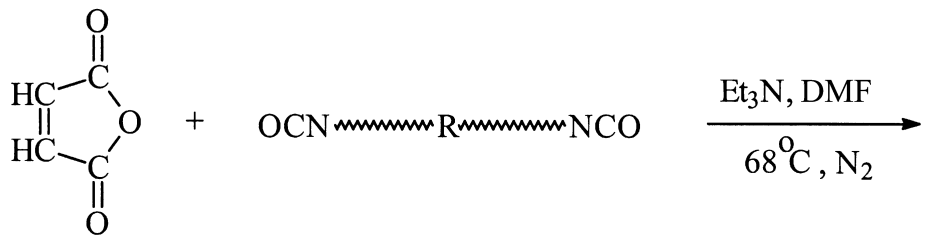

Maleic anhydride PU prepolymer<smiles>O=C1C=CC(=O)N1[C@H]1[C@H]2C=C[C@H](C2)[C@H]1O</smiles>

Table 1

Materials

\begin{tabular}{lll}
\hline Designation & Description & Source \\
\hline PPG400 & Poly(oxypropylene) glycol, MW $=400$ & Arcol Co., Taiwan \\
PPG1000 & Poly(oxypropylene) glycol, MW $=1000$ & Arcol Co., Taiwan \\
PPG2000 & Poly(oxypropylene) glycol, MW =2000 & Arcol Co., Taiwan \\
PBA700 & Poly(butylene adipate) glycol, MW $=700$ & Tai Gin Co., Taiwan \\
PBA1000 & Poly(butylene adipate) glycol, MW $=1000$ & Tai Gin Co., Taiwan \\
PBA2000 & Poly(butylene adipate) glycol, MW =2000 & Tai Gin Co., Taiwan \\
DGEBA & Diglycidyl ether of bisphenol A, EEW $=186, \mathrm{n}=0.113$ & Dow-Chemical Co., USA \\
MDI & 4,4'-Diphenyl methane diisocyanate, Eq. wt. $=125$ & BASF Wyandotte Co., Switzerland \\
TDMP & $2,4,6-$ Tri(dimethyl aminomethyl) phenol & Ciga-Geigy Co., Switzerland \\
DCPO & Dicumyl peroxide & Merck Co., Germany \\
\hline
\end{tabular}




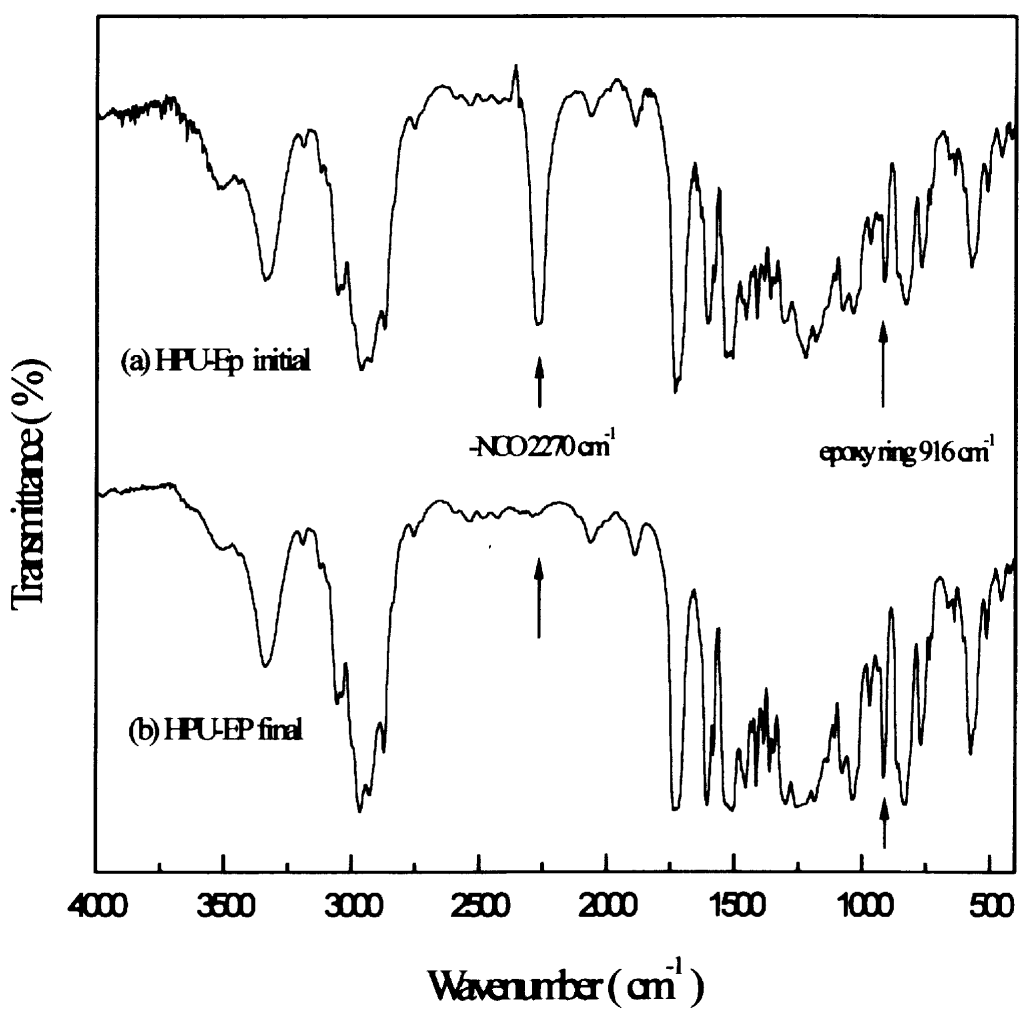

Fig. 2. FT-IR spectra during the reaction of HPU(PBA700) prepolymer with the hydroxyl group of DGEBA resin: (a) initial; (b) final.

The UBMI was obtained after removal of solvent by vacuum.

\subsection{Preparation of graft interpenetrating polymer network of UBMI and epoxy (UBMI/Ep graft-IPN)}

An appropriate amount of the epoxy resin (DGEBA) was mixed with the different contents of UBMI and then placed in a cup. Next, $10 \mathrm{wt} \%$ of the above PU graft agent, $3 \mathrm{phr}$ of curing agent (TDMP) and $1 \mathrm{wt} \%$ of initiator (DCPO) were added to the mixture. After mechanical agitation and degassing for $30 \mathrm{~s}$, the mixture was cast into an aluminum mold at $120^{\circ} \mathrm{C}$ and pressed for $1 \mathrm{~h}$. Finally, it was further heated to $180^{\circ} \mathrm{C}$; this temperature was maintained for another $2 \mathrm{~h}$. Upon removal from the mold, samples were stored in a desiccator, where the relative humidity was maintained at $50 \%$ for at least 3 days before testing.

\subsection{Testing methods}

The Fourier transform IR (FT-IR) analysis was performed on a BIO-RAD FTS-40 FTIR spectrophotometer at a resolution of $4 \mathrm{~cm}^{-1}$. The sample was placed directly on a $\mathrm{KBr}$ pellet.

Stress-strain properties were determined on a Tensilon (Mode: TCF-RC, Yashima works Ltd, Japan) test unit. The test procedure followed ASTM D-638, with a crosshead speed of $1 \mathrm{~cm} / \mathrm{min}$. The Izod impact strength of the graft-IPNs was measured according to ASTM D-256.

The fracture energy $G_{\mathrm{IC}}$ was measured using compacttension specimens (CTS) [14]. The $G_{\mathrm{IC}}$ value was calculated as follows:

$$
\begin{aligned}
G_{\mathrm{IC}}=[ & \left.Y^{2}(a / w) P^{2} a\right] E W^{2} b^{2} \\
Y(a / w)= & 29.6-186(a / w)+656(a / w)^{2}-1017(a / w)^{3} \\
& +639(a / w)^{4}
\end{aligned}
$$

Here $a, b$, and $E$ are crack length, thickness, and modulus, respectively, of the specimen, and $P$ is the load on the specimen. A sharp precrack was made with a razor blade before the test. The specimens were tested on a screw-driven Instron machine at a crosshead rate of $0.5 \mathrm{~cm} / \mathrm{min}$. The $\mathrm{G}_{\mathrm{IC}}$ value was determined by averaging values of $6-8$ specimens of the graft-IPNs.

Morphological studies were performed by using scanning electron microscopy (SEM). Microphotographs were taken of the surface made by fracturing the specimen in liquid nitrogen and then coating it with gold powder. 


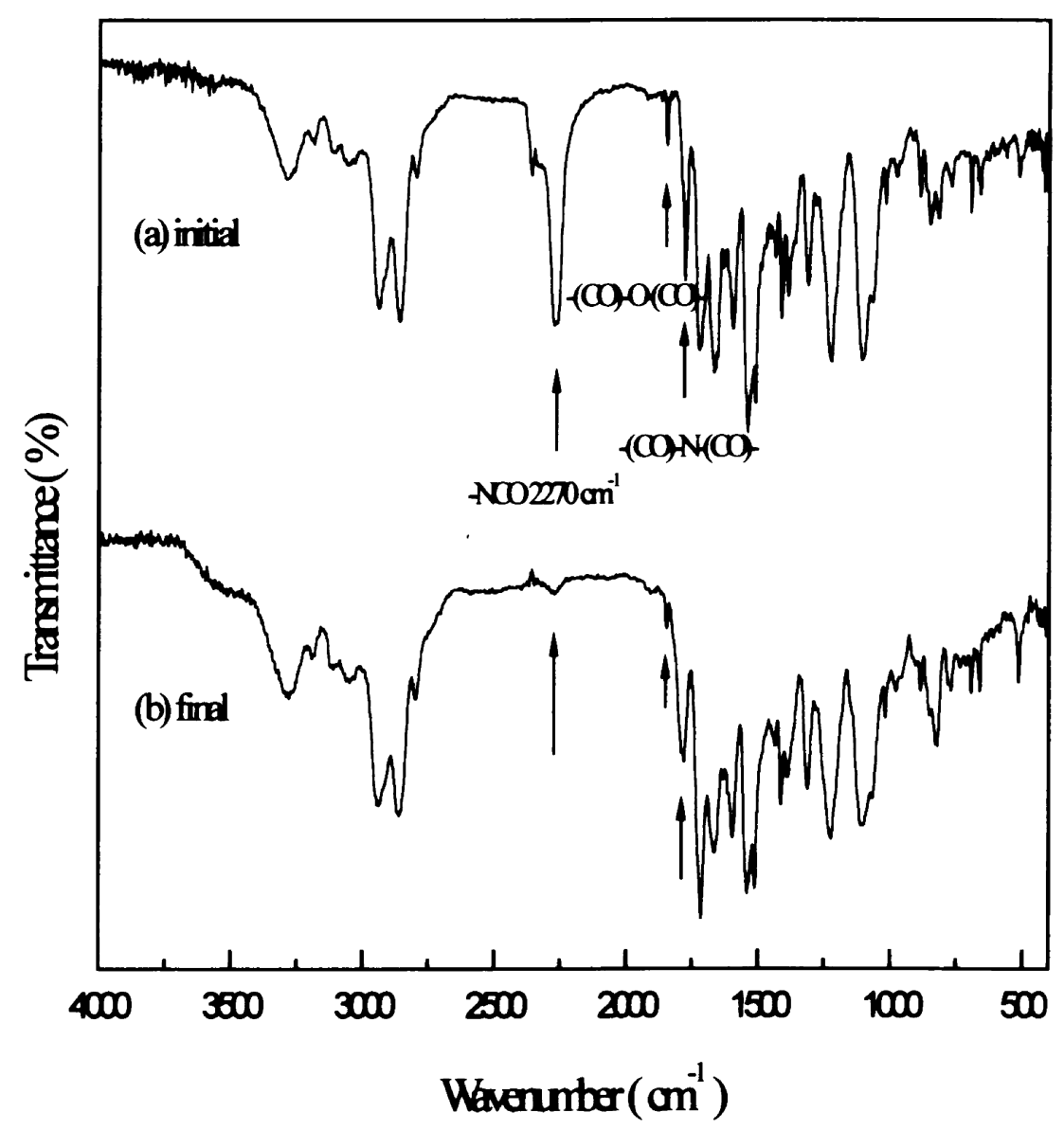

Fig. 3. FT-IR spectra during the reaction of PU prepolymer with maleic anhydride: (a) initial; (b) final.

\section{Results and discussion}

\subsection{FT-IR spectroscopy}

Since the aromatic $-\mathrm{CH}$ - group did not participate in the reaction between NCO-terminated PU prepolymer and hydroxyl group of 2-hydroxyethyl methacrylate (HEMA) (see Eq. (1)), the intensity of the IR absorption of the aromatic $-\mathrm{CH}-\left(2940 \mathrm{~cm}^{-1}\right)$ should not change during the reaction. Hence, the intensity ratio for the isocyanate peak $\left(2270 \mathrm{~cm}^{-1}\right)$ to the aromatic -CH- group $\left(2940 \mathrm{~cm}^{-1}\right) \mathrm{can}$ be used as an indication of the degree of reaction between NCO-terminated PU prepolymer and hydroxyl groups of HEMA. As shown in Fig. 1, the intensity ratio was high at the beginning of the reaction. As the reaction proceeded, it decreased until the hydroxyl groups in the HEMA had completely reacted with the isocyanate groups in the PU prepolymer. Thus, it was confirmed that the PU was reacted to the HEMA. The remaining isocyanate in the system were further reacted to form PU graft agent by the addition of epoxy (DGEBA) resin (see Eq. (2)). As shown in Fig. 2, when the PU prepolymer (included HEMA) was introduced into the epoxy (DGEBA) resin the absorption peak of the $-\mathrm{NCO}$ group at $2270 \mathrm{~cm}^{-1}$ reduced gradually in intensity with reaction time. Finally, the peak disappeared at a longer time. It indicates that the reaction between the remaining - $\mathrm{NCO}$ group of the PU prepolymer (included HEMA) and the pendent secondary hydroxyl group of epoxy (DGEBA) resin was completed. Thus, it was confirmed that the PU graft agent was obtained.

As shown in Fig. 3, the reaction between NCOterminated PU prepolymer and maleic anhydride (see Eq. (3)) was monitored by the disappearance of the characteristic peaks of isocyanate group $\left(2270 \mathrm{~cm}^{-1}\right)$ and acid anhydride $\left(1848 \mathrm{~cm}^{-1}\right)$ in IR spectra. The disappearance of strong and broad-NCO absorption peak at $2270 \mathrm{~cm}^{-1}$ and $\mathrm{C}=\mathrm{O}$ stretching vibration peak of acid anhydride at $1848 \mathrm{~cm}^{-1}$ in the IR spectra is evidence that the reaction has taken place between the PU prepolymer and maleic anhydride. Furthermore, a characteristic carbonyl absorption peak of imide was observed at $1784 \mathrm{~cm}^{-1}$. Another, the elemental analysis of the products also gave another evidence for the formation of an imide group after the evolution of the stoichiometric amount of $\mathrm{CO}_{2}$. 


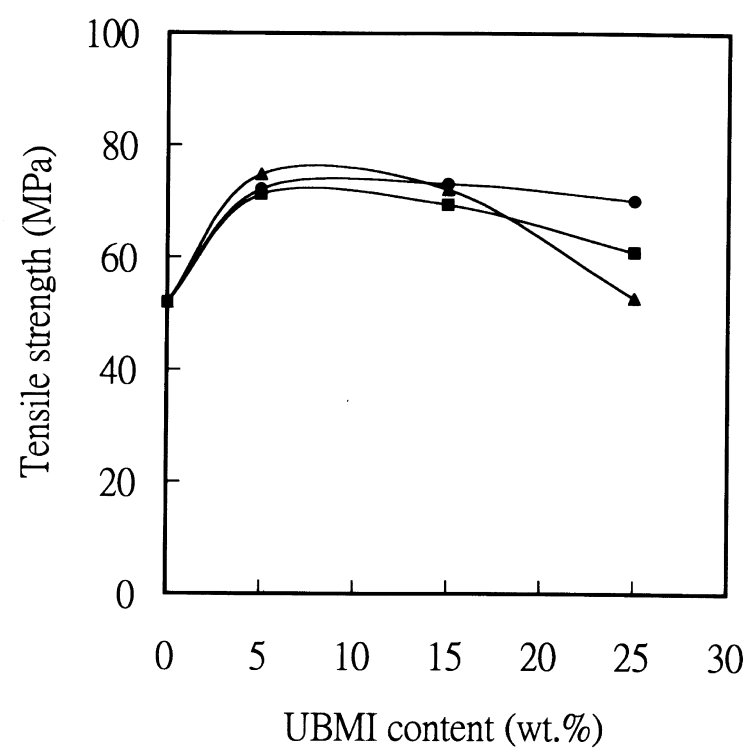

Fig. 4. Variations of tensile strength with UBMI content in UBMI/Ep graftIPNs for the PU(PBA) graft agent based on PU(PBA700) PU(PBA1000) (๑), PU(PBA2000) ( $\mathbf{\Delta})$.

\subsection{Stress-strain properties}

Figs. 4 and 5 showed the mechanical properties of the graft interpenetrating polymer network of UBMI and epoxy (UBMI/Ep graft-IPNs) by $10 \mathrm{wt} \% \mathrm{PU}$ grafting agent. The tensile strength of both kinds of UBMI/Ep graft-IPNs, those based on PU(PBA) grafting agent (Fig. 4) and PU(PPG) grafting agent (Fig. 5), increased with

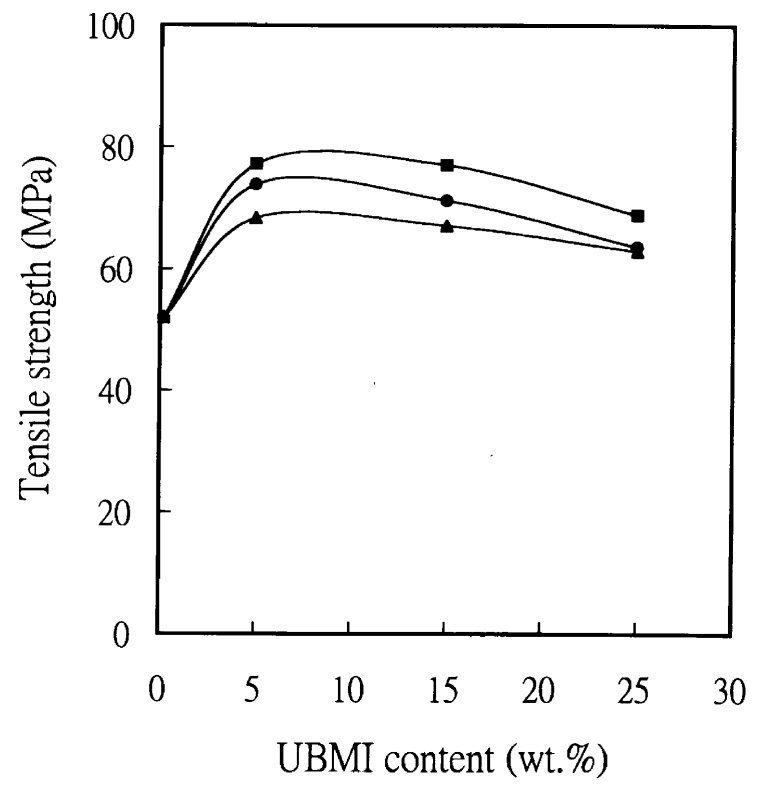

Fig. 5. Variations of tensile strength with UBMI content in UBMI/Ep graftIPNs for the PU(PPG) graft agent based on PU(PPG400) PU(PPG1000) (•), PU(PPG2000) (ム).

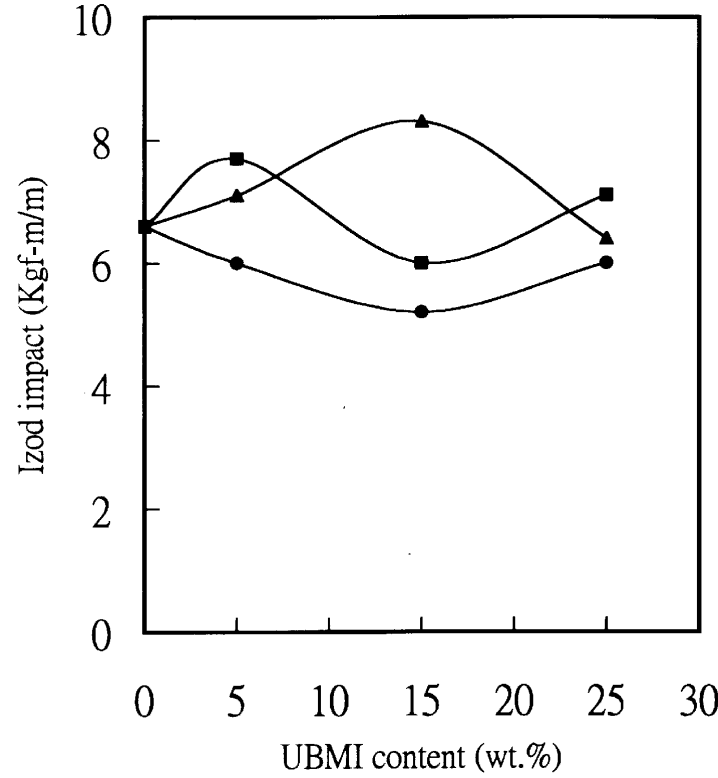

Fig. 6. Variations of Izod impact strength with UBMI content in UBMI/Ep graft-IPNs for the PU(PBA) graft agent based on PU(PBA700) PU(PBA1000) (๑), PU(PBA2000) ( $\mathbf{\Delta})$.

increasing UBMI content up to a maximum value, then decreased with the continued increase of UBMI in graftIPNs with PU graft agent. The maximum tensile strength occurred at UBMI/epoxy weight ratios between 5/95 and $15 / 85$. This improvement in tensile strength resulted from the increased degree of interpenetrating as a result of the graft structure, but decreased by introducing the softer segment of the UBMI. Therefore, a maximum value in tensile strength, which was the balanced point of these

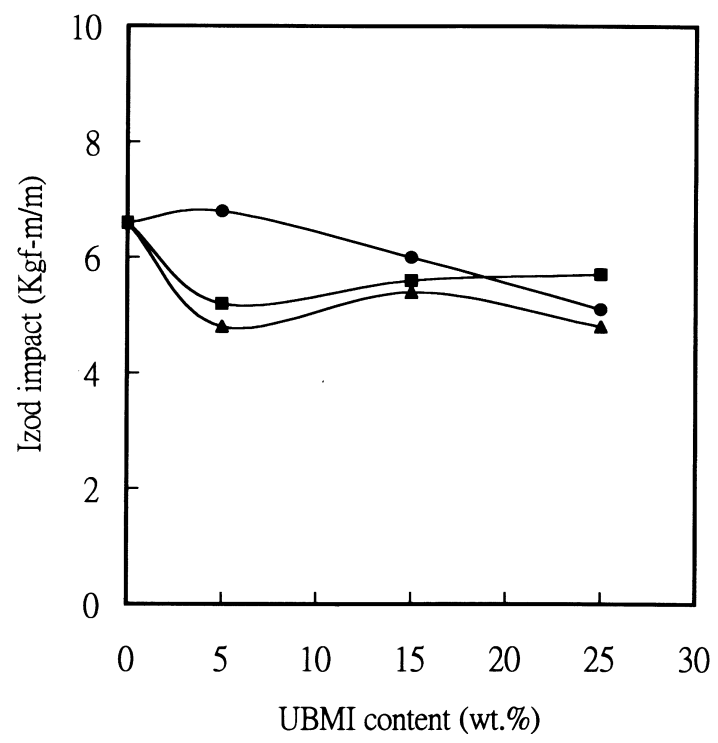

Fig. 7. Variations of Izod impact strength with UBMI content in UBMI/Ep graft-IPNs for the PU(PPG) graft agent based on PU(PPG400) (ם), PU(PPG1000) (•), PU(PPG2000) ( $\mathbf{\Delta})$. 


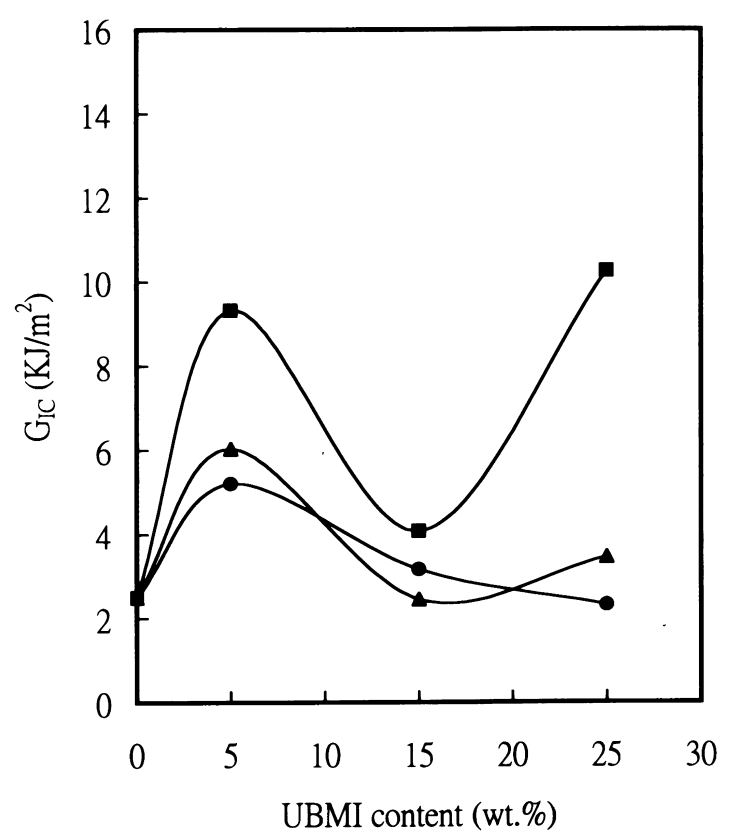

Fig. 8. Variations of fracture energy $G_{\text {IC }}$ with UBMI content in UBMI/Ep graft-IPNs for the PU(PBA) graft agent based on PU(PBA700) (ם), PU(PBA1000) (•), PU(PBA2000) (ム).

two contributions. It was also observed that the UBMI/Ep graft-IPNs with shorter soft graft chain in the PU (i.e. PPG400 or PBA700) had higher tensile strength than those with longer graft chains in the PU (i.e. PPG2000 or PBA2000). The reason for this is the increased crosslinking in the UBMI/Ep graft-IPNs with shorted soft graft chain.

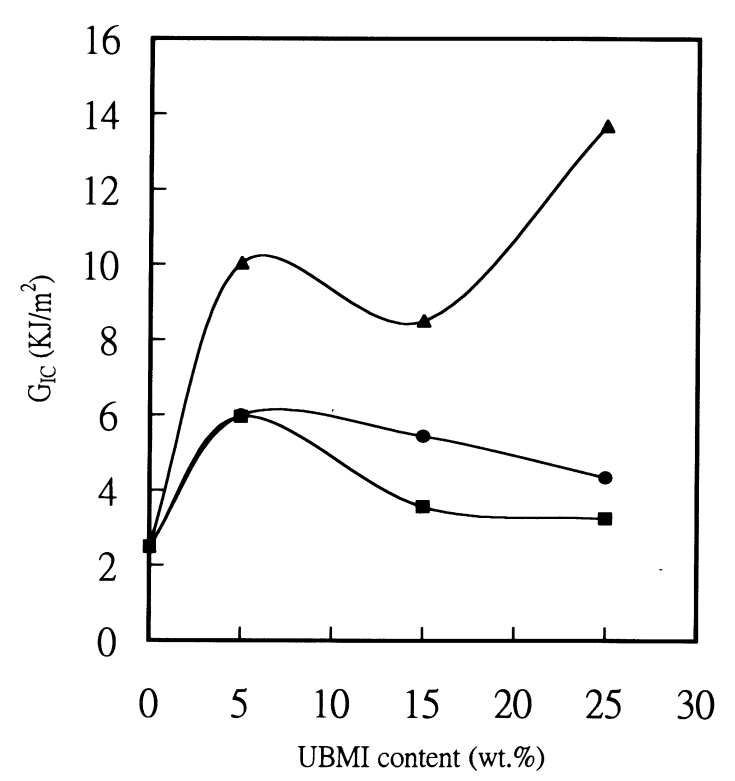

Fig. 9. Variations of fracture energy $G_{\mathrm{IC}}$ with UBMI content in UBMI/Ep graft-IPNs for the PU(PPG) graft agent based on PU(PPG400) (ם), PU(PPG1000) (•), PU(PPG2000) (ム).

\subsection{Izod impact strength}

The Izod impact strength of the UBMI/Ep graft-IPNs with $10 \mathrm{wt} \%$ PU graft agent based on PPG [PU(PPG)] and on PBA [PU(PBA)] is shown in Figs. 6 and 7, respectively. In previous articles, the cured UBMI has rubbery property $[10,11]$ and the cured epoxy has rigidity property $[5-9,15]$. For both kinds of PU graft agent with various molecular weight in the UBMI/Ep graft-IPNs, the Izod impact strength increased with the UBMI contents increasing. This behavior is a result of the interpenetrating network effect, in addition to the grafted structure between UBMI and epoxy by PU graft agent. The UBMI/Ep graft-IPNs based on PU(PBA) graft agent of better compatibility than those based on PU(PPG) graft agent, show a homogeneous morphology (Fig. 10), while the graft agent PU(PPG)-based UBMI/Ep graft-IPNs were all heterogeneous (Fig. 11). The better compatibility of graft agent PU(PBA)-based UBMI/Ep graft-IPNs led to more interpenetration and results in higher impact strength.

\subsection{Fracture energy $\left(G_{I C}\right)$}

The fracture energy $G_{\text {IC }}$ of UBMI/Ep graft-IPNs with $10 \mathrm{wt} \%$ PU graft agent based on PU(PPG) and on $\mathrm{PU}(\mathrm{PBA})$ are shown in Figs. 8 and 9, respectively. As mentioned previously $[5-9,16,17]$, the toughness value of epoxy resin for the fracture energy $G_{\mathrm{IC}}$ (i.e. low shear rate fracturing test) is different from the Izod impact strength (i.e. high shear rate fracturing test). However, to increase the fracture energy $G_{\text {IC }}$ value, the formation of a heterogeneous morphology with sufficient rubber domains distributed in the matrix is more important.

As shown in Fig. 8, the fracture energy $G_{\text {IC }}$ of UBMI/Ep graft-IPNs with PU(PBA) graft agent increased with increasing UBMI content up to a maximum value, then decreased with the continued increase of UBMI in graftIPNs. The maximum fracture energy $G_{\mathrm{IC}}$ occurred at UBMI content $5 \mathrm{wt} \%$. The increase in $G_{\mathrm{IC}}$ was probably attributed to the increase of toughness of the matrix from the increased degree of interpenetration as a result of the graft structure. The microscopic structure of UBMI/Ep graft-IPNs with PU(PBA) graft agent as shown in Fig. 10, illustrated that they are in a homogeneous morphology with good compatibility between the UBMI and epoxy (DGEBA).

On the other hand, the UBMI/Ep graft-IPNs with PU(PPG) graft agent have a significant increase in fracture energy $G_{\mathrm{IC}}$, as the UBMI content increases (see Fig. 9). From the microphotographs shown in Fig. 11 the UBMI dispersed particles in the epoxy (DGEBA) matrix can be seen. This two-phase morphology had been proved to serve effectively the toughening mechanism as mentioned before [5-9,16,17]. In other words, the UBMI/Ep graftIPNs with PU(PPG) graft agent system has higher fracture energy than UBMI/Ep graft-IPNs with PU(PBA) graft agent 


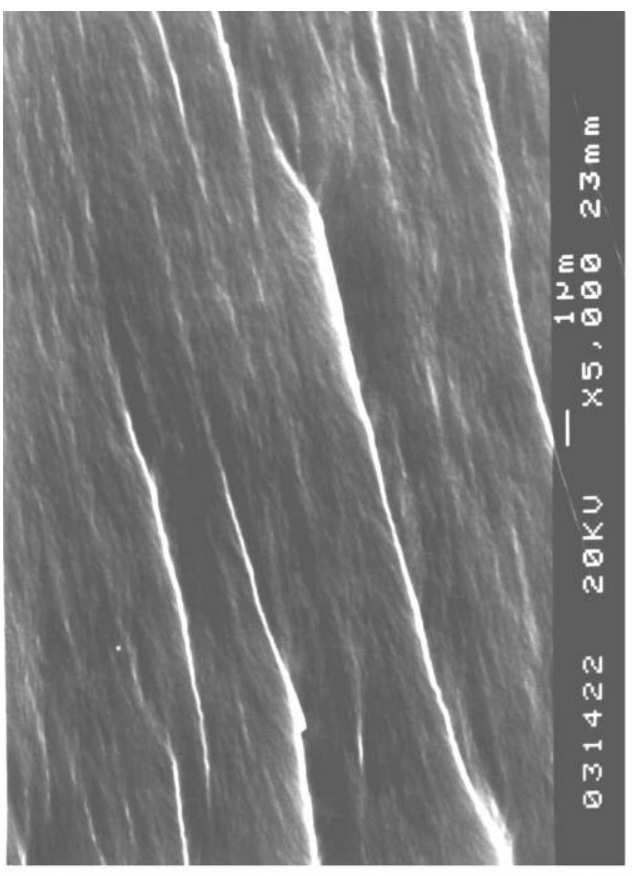

(a)

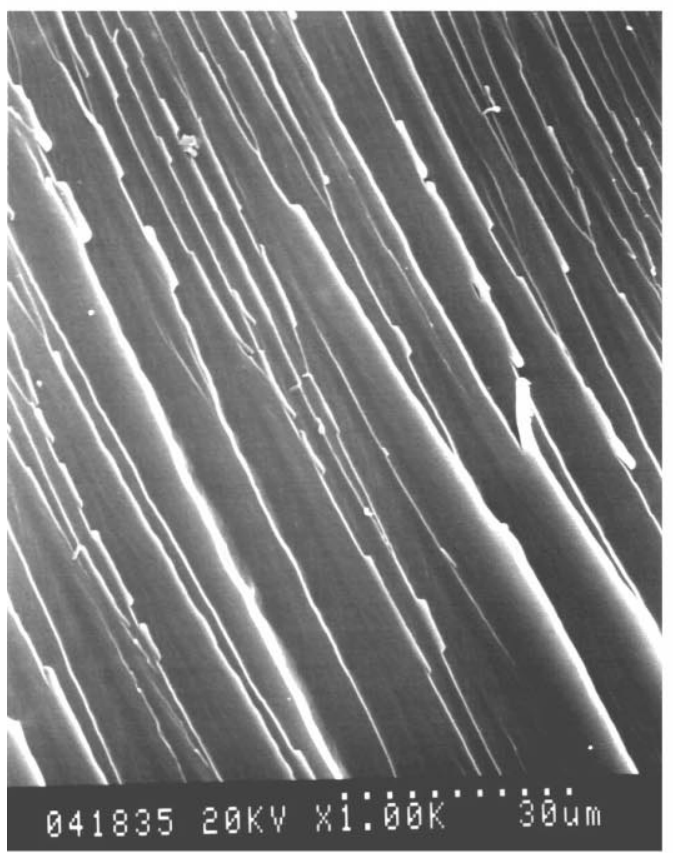

(c)

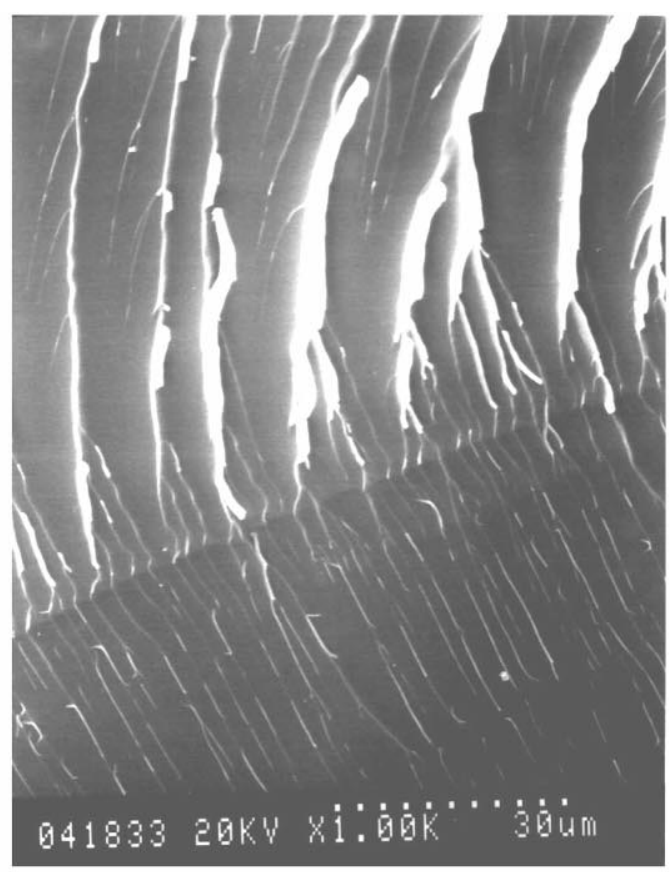

(b)

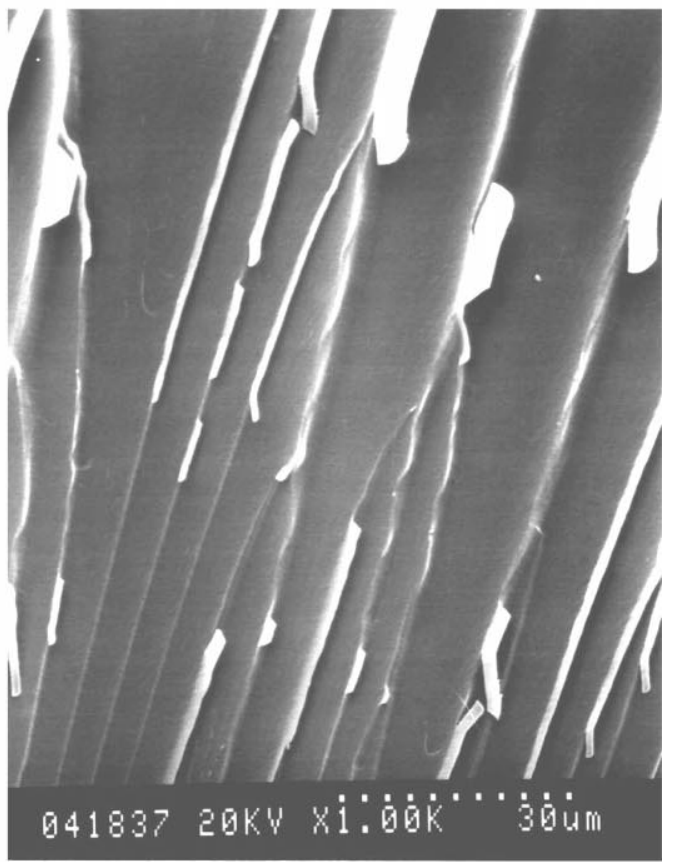

(d)

Fig. 10. Scanning electron photomicrographs for UBMI/Ep graft-IPNs with PU(PBA) graft agent based on PU(PBA2000) at various UBMI/Ep ratio: (a) 0/100; (b) $5 / 95$; (c) $15 / 85$; (d) $25 / 75$.

system (Figs. 8 and 9). It was also observed that the UBMI/ Ep graft-IPNs with longer soft graft chains in the PU (i.e. PPG400 or PBA700) had higher fracture energy $G_{\mathrm{IC}}$ than those with shorter graft chains in PU (i.e. PPG2000 or PBA2000). The reason for this is the increased toughness in the matrix with longer soft graft chains.

\subsection{Compatibility and morphology}

The microphotographs of the UBMI/Ep graft-IPNs with PU(PBA2000) graft agent are shown in Fig. 10. They show a homogeneous microstructure. The fracture surface shows the roughness increased as the UBMI content grew. It means 


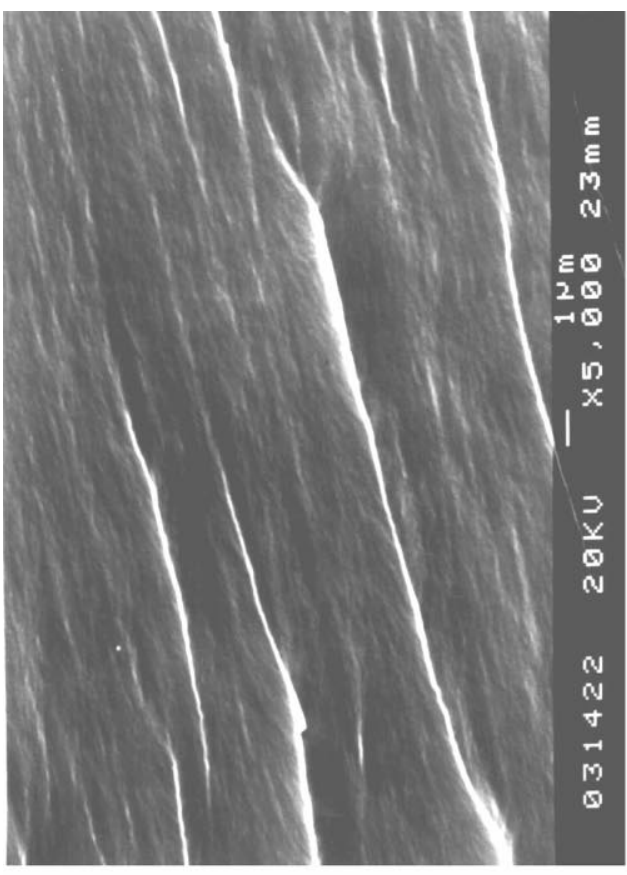

(a)

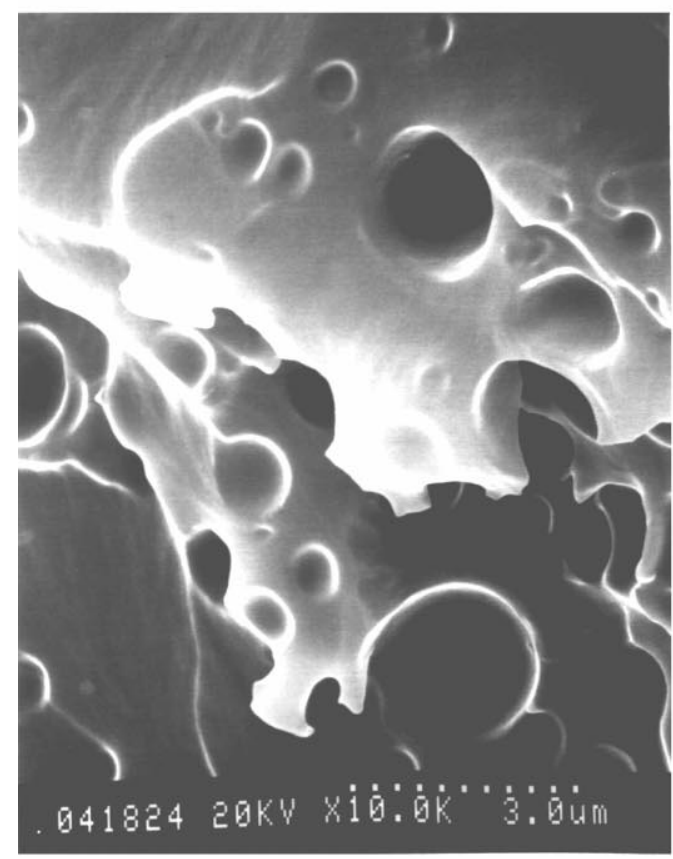

(c)

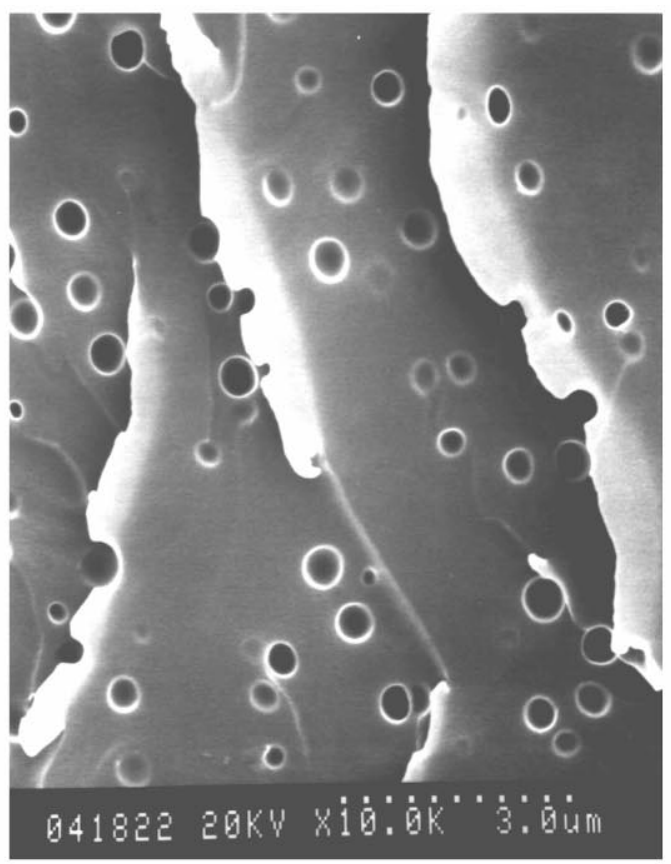

(b)

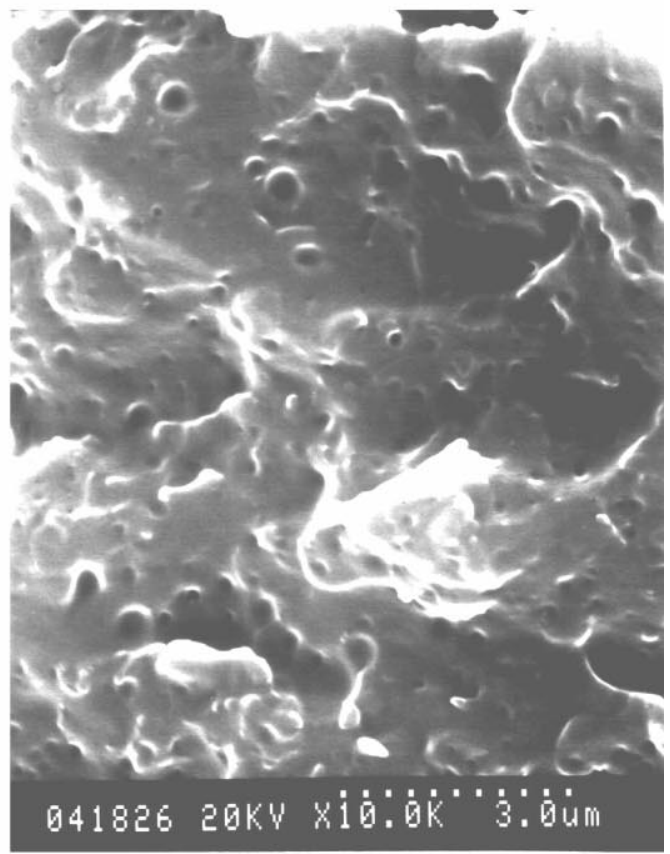

(d)

Fig. 11. Scanning electron photomicrographs for UBMI/Ep graft-IPNs with PU(PPG) graft agent based on PU(PPG2000) at various UBMI/Ep ratio: (a) 0/100; (b) $5 / 95$; (c) $15 / 85$; (d) $25 / 75$.

that the UBMI/Ep graft-IPNs with PU(PBA) graft agent have good compatibility between the UBMI and epoxy (i.e. DGEBA). It was also observed that the fracture surface have many "beach line" existing. And the number of "beach line" increased with increasing UBMI content. In contrast, the UBMI is incompatible with the epoxy (DGEBA) by the polyether-type PU(PPG) graft agent. It shows that the UBMI/Ep graft-IPNs with PU(PPG2000) graft agent has a phase-separated microstructure as shown in Fig. 11. The UBMI particles are dispersed in the epoxy (DGEBA) matrix. As shown in Fig. 11(b) and (c), the size of UBMI particles increased with the growth of the UBMI content. 
The round particles disappeared and a rough surface is found in Fig. 11(d) when the UBMI content increased up to $25 \mathrm{wt} \%$. This is an indication for two continuous phases within the region of the phase inversion.

\section{Conclusion}

The tensile strength of the UBMI/Ep graft-IPNs with PU graft agent increased with growing amount of UBMI incorporated in the epoxy (DGEBA), but it went down when the amount of UBMI had reached beyond a certain level. The maximum tensile strength was the balance point of the contributions from the interpenetrating effect and the PU soft segment character. The UBMI/Ep graft-IPNs with PU(PBA) graft agent toughened the epoxy (DGEBA) matrix, thus it shows higher Izod impact strength than the UBMI/Ep graft-IPNs with PU(PPG) graft agent. On the contrary, the UBMI/Ep graft-IPNs with PU(PPG) graft agent system which has UBMI particle dispersion in epoxy (DGEBA) matrix shows higher fracture energy $G_{\mathrm{IC}}$ than the UBMI/Ep graft-IPNs with PU(PBA) graft agent. The UBMI/Ep graft-IPNs with PU graft agent produced different morphological microstructures depending on the types of the polyols used in PU graft agent. The UBMI/Ep graft-IPNs with the polyester-type PU graft agent may result in a homogeneous phase, while the UBMI/Ep graft-IPNs with the polyether-type PU graft agent presents a heterogeneous phase with UBMI particles dispersed in epoxy (DGEBA) matrix.

\section{Acknowledgements}

The authors would like to thank the National Science Council, Taipei, Taiwan ROC for financially supporting this research under Contract No. NSC 86-2745-E-002$014 \mathrm{R}$.

\section{References}

[1] Sperling LH, Friedmen DW. J Polym Sci A-2 1970;7:425.

[2] Frisch HL, Klemper D, Frisch KC. J Polym Sci B 1969;7:775.

[3] Huelck V, Thomas DA, Sperling LH. Macromolecules 1972;5:340.

[4] Frisch KC, Klempner D, Mukherjes SK. J Appl Polym Sci 1974;18:689.

[5] Hsieh KH, Han JL. J Polym Sci, Polym Phys Ed 1990;28:623.

[6] Hsieh KH, Han JL. J Polym Sci, Polym Phys Ed 1990;28:783.

[7] Han JL, Chern YC, Li KY, Hsieh KH. J Appl Polym Sci 1998;70:529.

[8] Han JL, Li KY. J Appl Polym Sci 1998;70:2635.

[9] Han JL, Li KY. Polym J 1999;31(5):401.

[10] Liao DC, Hsieh KH. J Polym Sci, Polym Chem 1994;32:1665.

[11] Liao DC, Hsieh KH, Kao SC. J Polym Sci, Polym Chem 1995;33:481.

[12] Thomas DA, Spering LH. Polymer blend, vol. 2. New York: Academic, 1978

[13] Hepburn C. Polyurethane elastomer. Barking: Applied Science Publishers, 1982 (p. 280).

[14] Ting RY, Cottington RL. J Appl Polym Sci 1980;25:1815.

[15] Lee H, Neville K. Handbook of epoxy resin. New York: McGraw Hill, 1967.

[16] Han JL, Tseng SM, Mai JH, Hsieh KH. Die Angew Makromol Chem 1990;182:193.

[17] Han JL, Tseng SM, Mai JH, Hsieh KH. Die Angew Makromol Chem 1991;184:89. 